Portland State University

PDXScholar

Systems Science Faculty Publications and

Presentations

Systems Science

$10-2017$

\title{
Formalizing the Panarchy Adaptive Cycle with the Cusp Catastrophe
}

\author{
Martin Zwick \\ Portland State University, zwick@pdx.edu \\ Joshua Hughes
}

Follow this and additional works at: https://pdxscholar.library.pdx.edu/sysc_fac

Part of the Logic and Foundations of Mathematics Commons, and the Mathematics Commons Let us know how access to this document benefits you.

\section{Citation Details}

Zwick, M. and Hughes, J. (2017). Formalizing the Panarchy Adaptive Cycle with the Cusp Catastrophe. [Post-print] Proceedings of the 2017 International Conference of The Computational Social Science Society of the Americas. Article No.: 13 Pages 1-5

This Post-Print is brought to you for free and open access. It has been accepted for inclusion in Systems Science Faculty Publications and Presentations by an authorized administrator of PDXScholar. Please contact us if we can make this document more accessible: pdxscholar@pdx.edu. 


\section{Formalizing the Panarchy Adaptive Cycle with the Cusp Catastrophe}

\author{
Martin Zwick \\ Systems Science \\ Portland State University \\ Portland OR 97207-0751 USA \\ zwick@pdx.edu
}

\begin{abstract}
The panarchy adaptive cycle, a general model for change in natural and human systems, can be formalized by the cusp catastrophe of René Thom's topological theory. Both the adaptive cycle and the cusp catastrophe have been used to model ecological, economic, and social systems in which slow and small continuous changes in two control variables produce fast and large discontinuous changes in system behavior. The panarchy adaptive cycle, the more recent of the two models, has been used so far only for qualitative descriptions of typical dynamics of such systems. The cusp catastrophe, while also often employed qualitatively, is a mathematical model capable of being used rigorously. If the control variables from the adaptive cycle are taken as parameters in the equation for the cusp catastrophe, a cycle very similar to the adaptive cycle can be constructed. Formalizing the panarchy adaptive cycle with the cusp catastrophe may provide direction for more rigorous applications of the adaptive cycle, thereby augmenting its usefulness in guiding sustainability efforts.
\end{abstract}

\section{KEYWORDS}

panarchy, adaptive cycle, cusp catastrophe, catastrophe theory, ecological and evolutionary systems, sustainability

\section{ACM Reference format:}

Martin Zwick and Joshua Hughes, 2017.

https://doi.org/0000001.0000001

\section{INTRODUCTION}

The panarchy adaptive cycle [1] is a model for ecological systems intended as a step toward a theory to guide sustainability efforts. The model is based on the idea that ecological systems change qualitatively over time in certain regular patterns. The adaptive cycle models the relations between slow and fast, large and small, and continuous and discontinuous ecological processes. Because it is based on general ideas, the model has been applied also to human, i.e., economic and social, systems. However, so far the adaptive cycle does not seem to have been developed beyond a qualitative description of dynamic patterns that is used to classify systems, characterize events, or suggest questions and hypotheses. Gunderson and Holling themselves state that they "do not know

\author{
Joshua Hughes \\ Adidas \\ 5055 N Greeley Avenue \\ Portland, OR 97217 USA \\ Joshua.Hughes@adidas.com
}

the minimal mathematical properties necessary for a model to generate adaptive cycles"(p.178) [1, p.178].

In this paper, we show that the adaptive cycle can be formalized with catastrophe theory, specifically with the cusp catastrophe. Formalizing the adaptive cycle model does not operationalize it by indicating how variables in the model are to be measured. Nor does formalization justify the model theoretically or empirically. It merely shows that the model can be expressed precisely. There may be other ways to formalize the adaptive cycle. Still, the use of catastrophe theory, and specifically the cusp catastrophe, for ecological modeling is extensive [2-13] and Holling [14-15] himself has used it for this purpose, so applying it to the adaptive cycle could be promising. However, to the best of our knowledge, no one yet has applied the cusp catastrophe to the adaptive cycle itself.

\section{THE ADAPTIVE CYCLE}

The adaptive cycle tracks "potential" which changes as a function of two orthogonal variables defined as "connectedness" and "resilience." Potential is a measure of capital appropriate for the system, for example biomass in an ecological system or assets in an economic system. Connectedness is the pervasiveness and strength of the network of internal relations, and thus the degree to which they have greater influence on the system than external factors. Resilience is usually defined as the system's ability to remain in the same basin of attraction despite disturbances, where this ability depends on the basin size, the closeness of the attractor to the basin boundaries, and other related properties of the dynamic system. For resilience to be a variable similar to connectedness it should really be defined in terms of these dynamic properties, and the "ability to remain in the same basin," which is a measure of system behavior, should be regarded as a surrogate for these properties.

As shown in Fig. 1, from an oblique view, the adaptive cycle resembles a horizontal figure 8 , the symbol for infinity, though this shape is not visible from other viewpoints. As connectedness and resilience change, potential rises and falls through the four phases labeled as the r-phase, K-phase, $\Omega$-phase, and $\alpha$-phase. The cycle thus consists of the logistic growth curve, composed of the familiar $\mathrm{r}$ and $\mathrm{K}$ phases, augmented with two additional phases, namely $\Omega$ and $\alpha$. This is a symbolic diagram in which time is not a horizontal axis but rather motion along the cycle (indicated by the arrows in Fig. 1) occurring at different speeds. 


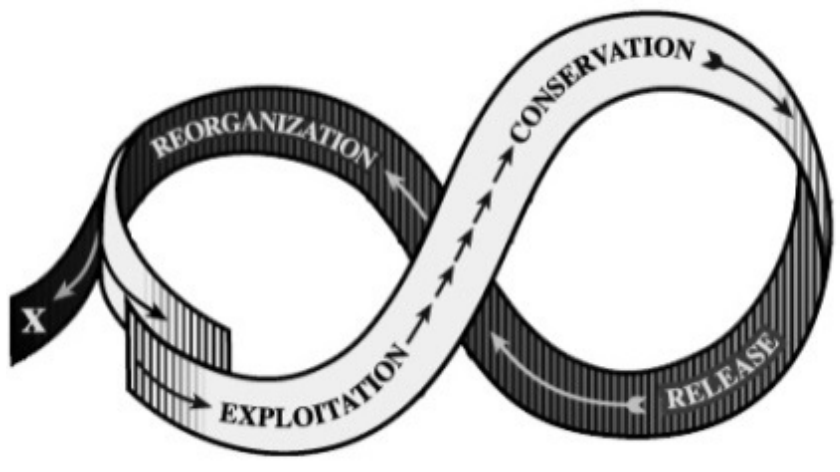

Figure 1: Four phases of the adaptive cycle: exploitation $(\mathbf{r})$, conservation $(\mathrm{K})$, release $(\Omega)$, reorganization $(\alpha)$.

The adaptive cycle begins with the r-phase, the exploitation or growth phase, in which agents in the system scramble for resources, and those that grow fastest survive. During exploitation connectedness, resilience, and potential all increase. Communities of agents begin to self-organize, i.e., agents develop relationships with other agents that improve their competitiveness, but as connections increase the dominant agents begin to exert more control. The system then transitions to the K-phase, or conservation phase, in which connectedness increases, growth slows, potential peaks, and resilience decreases. As the K-phase continues, the system becomes overconnected and thus increasingly rigid and vulnerable. Eventually a disturbance triggers the $\Omega$-phase-release or creative destruction-and this release causes potential to drop precipitously. But as the old connections break down, new interactions occur; these weak interactions represent innovation and experimentation within the system. While many experiments fail, others do not, and the loss of connectedness allows potential and resilience to increase once again, as the system enters the $\alpha$-phase of reorganization and renewal. Reorganization involves further changes, so not all successful experiments remain in the system. By the end of the $\alpha$-phase some potential is lost (the cycle is "leaky" at this point), and the cycle begins anew.

The first two phases ( $\mathrm{r}$ and $\mathrm{K}$ ) comprise the front loop, or the slow, predictable production and accumulation that lead to stability; the third and fourth phases $(\Omega$ and $\alpha)$ comprise the back loop, or the fast and unpredictable innovation and reorganization that constitute adaptation. At this general level of explanation it seems apparent that the adaptive cycle might be applied to many different natural and human systems with some success.

\section{THE CUSP CATASTROPHE}

The panarchy adaptive cycle bears some obvious similarities to the cusp catastrophe [16] [17]. All cusp catastrophe models include a state or behavior variable that changes relative to two orthogonal control variables and system behavior marked by the possibility of abrupt and discontinuous change [17]. As shown in Fig. 2, the behavior surface is a folded topological surface that can be visualized above a planar control surface. Any trajectory on the control surface causes a trajectory on the behavior surface directly above it. The behavior surface represents the equilibrium states for the system for all pairs of values of the control variables. For most control variable values there exists only one equilibrium state, but within the bifurcation zone, where the behavior surface overlaps itself, there are three equilibrium states: two stable and one unstable. The stable equilibria (attractors) define the upper and lower behavior sheets; the unstable equilibria (repellors) define the middle sheet. Because two stable states exist within the bifurcation zone, the state is determined by the history of the system, i.e., by the direction from which the bifurcation zone was entered. The point at which the three sheets meet is the cusp singularity. Control variables for the cusp can be defined either as (i) opposing ("conflicting") factors, where one variable tends to move the state to the upper sheet and the other to the lower sheet, or as (ii) as normal and splitting factors, which result from a 45-degree rotation of the conflicting factors, where the splitting factor (directed along the sum of conflicting factors) determines whether or not a catastrophic jump is possible (it is possible forward of the singularity) and, if so, how large the jump is, while $\mathrm{t}^{-\ldots \ldots \ldots+1}$ between conflicti
stem to either the lower sheet

Figure 2: Cusp catastrophe with behavior surface above control surface with two opposing control factors $c_{1}$ and $\mathbf{c}_{2}$.

As discussed above, the adaptive cycle also has two control variables, a state variable capable of discontinuous change, and a trajectory on a control surface that causes a trajectory on a behavior surface. It thus seems likely that the adaptive cycle can be formalized by the cusp catastrophe. To investigate this proposition, the adaptive cycle was constructed using the equation for the cusp behavior surface. Specifically, the state variable, potential, was generated from the control variables, 
connectedness and resilience, which change over time in a closed (arbitrarily elliptical) trajectory. The sections that follow describe the method of constructing the adaptive cycle with the cusp and the results of this construction, a discussion of these results, and conclusions.

\section{MODELING THE ADAPTIVE CYCLE WITH THE CUSP CATASTROPHE}

The adaptive cycle is generated by the temporal relationship between connectedness and resilience. When viewed down the potential axis, these control variables follow a two-dimensional cycle resembling an ellipse, along which the system moves from $\mathrm{r}$ to $\mathrm{K}$ to $\Omega$ to $\alpha$ to $\mathrm{r}$ again, as shown in Fig. 3(a). If the precipitous drop in potential from the K-phase to the $\Omega$-phase is to occur at the correct point in the trajectory, connectedness and resilience should be oriented as opposing factors on the cusp control surface with a counterclockwise trajectory, as shown in Fig. 3(b). As opposing factors, connectedness tends to move behavior toward the upper sheet, and vulnerability (the opposite of resilience) tends to move behavior toward the lower sheet. In Fig. 3(b), the loss of potential in going from $\mathrm{K}$ to $\Omega$ is very distinct, as characterized by [1]; however, the loss of potential between the $\alpha$-phase and $r$-phase is not shown. Given the comparatively short time spans of the $\Omega$ and $\alpha$ phases when compared to the $\mathrm{r}$ and $\mathrm{K}$ phases, it seems unlikely that potential loss in the $\alpha$-phase can be as significant as the $\mathrm{K}$ to $\Omega$ potential loss. Yet the second drop in potential can also be represented on the cusp, as discussed below. (a) Adaptive cycle control factors

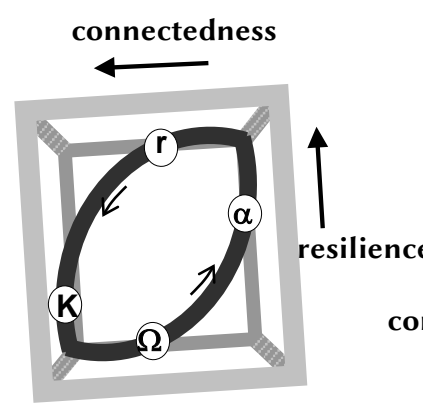

(b) Cusp catastrophe control factors

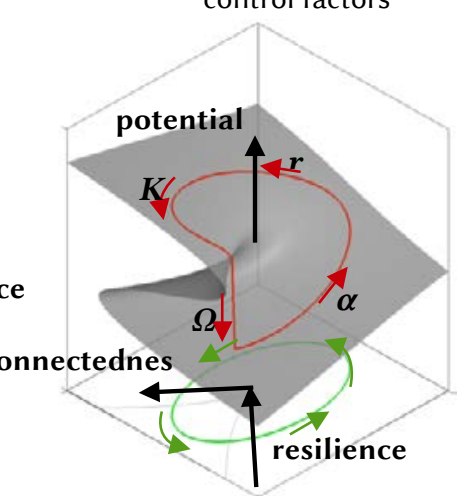

Figure 3: The orientation of control factors in (a) is used for (b), which also shows the vertical state variable, potential. (a) is tilted to avoid confusing the control axis of resilience with the vertical axis of potential.

The trajectory on the control surface of the cusp catastrophe can be specified by the parametric equations for an ellipse centered at an origin coincident with the singularity. With appropriate parameters the control trajectory can be shaped and oriented approximately consistent with the adaptive cycle.

The cusp catastrophe applies to gradient systems that maximize or minimize some "potential function," V,

$$
\mathrm{V}=1 / 4 \mathrm{P}^{4}-\mathrm{nP}-1 / 2 \mathrm{SP}^{2}
$$

where $\mathrm{P}$ is the state variable of the dynamic system (for the adaptive cycle, potential) and $\mathrm{n}$ and $\mathrm{s}$ are the normal and splitting factors, now taken to be the control variables. It is critical not to confuse the two different uses of the word "potential": $\mathrm{V}$ is the potential function that governs the dynamics, while $\mathrm{P}$ is the state variable of the dynamic system, which, when applied to the adaptive cycle, is the variable, potential. For gradient dynamics,

$$
\mathrm{dP} / \mathrm{dt}=\mathrm{kdV} / \mathrm{dP}
$$

where $\mathrm{k}$ is a constant. The behavior surface is given by $\mathrm{dP} / \mathrm{dt}=$ 0 ,

$$
\mathrm{P}^{3}-\mathrm{n}-\mathrm{sP}=0
$$

The normal and splitting factors $\mathrm{n}$ and s needed for (1) and (3) are obtained by a simple transformation of the two opposing factors, connectedness $(\mathrm{C})$ and resilience $(\mathrm{R})$ :

$$
\mathrm{n}=(\mathrm{C}+\mathrm{R}) / \sqrt{2} \quad \mathrm{~s}=(\mathrm{C}-\mathrm{R}) / \sqrt{2}
$$

Note that resilience is the negative of a conflicting factor, so the normal factor is aligned in the direction of the difference between the conflicting factors, while the splitting factor is aligned in the direction of their sum.

Closely associated with the adaptive cycle is the idea of panarchies, or nested adaptive cycles that interact across different time and space scales. Cusp catastrophes at different scales can be similarly nested, but this hierarchical aspect of the adaptive cycle is outside the scope of this paper.

\section{POTENTIAL LOSS FROM $\alpha$ PHASE TO r PHASE}

A final consideration is how to represent a loss of potential that may occur during the transition between the $\alpha$-phase and r-phase. This consideration might be considered optional, since the primary potential loss in the adaptive cycle is the transition from the K-phase to the $\Omega$-phase. However, since the adaptive cycle literature does speak about this second loss, we address this possibility in what follows.

For an elliptical trajectory centered about the singularity, only a single drop in potential can be represented with the canonical form of the cusp catastrophe. One can imagine at least two possible ways to model the loss of potential between the $\alpha$ and $r$ phases. The first is to deform the behavior surface in a way that encompasses this loss of potential. This can be accomplished by a transformation of the normal factor in Equation 3 from $n$ to $n^{\prime}$, where $n^{\prime}=n(1+s)$, resulting in Equation 5:

$$
\mathrm{P}^{3}-\mathrm{n}^{\prime}-\mathrm{sP}=0
$$

The second approach, using the same transformation of $n$ to $n$ ', does not require deformation of the behavior surface, but applies the transformation to the control surface trajectory. Representing the $\alpha$ to $r$ loss in potential with either approach is ad hoc, but the loss is not well enough characterized in the adaptive cycle literature to provide clear guidance on this matter. 
However, because the second approach changes the trajectory from what is usually specified for the adaptive cycle, the first approach is preferable and is the one presented here.

The constructed adaptive cycle, using the above transformation of the behavior surface equation, is shown in Fig. 4. The cycle is plotted on the behavior surface rotated in such a way that the view is similar to the view of the adaptive cycle shown in Fig. 1. The resulting cycle is similar to the cycle in that figure. It shows (i) the potential increasing during the r-phase as connectedness increases and then leveling off as resilience decreases during the K-phase, (ii) the catastrophic drop in potential from the K-phase to the $\Omega$-phase, (iii) the decreasing connectedness and increasing potential and resilience during the $\alpha$-phase, and (iv) the loss of potential during the transition from the $\alpha$-phase to the r-phase.

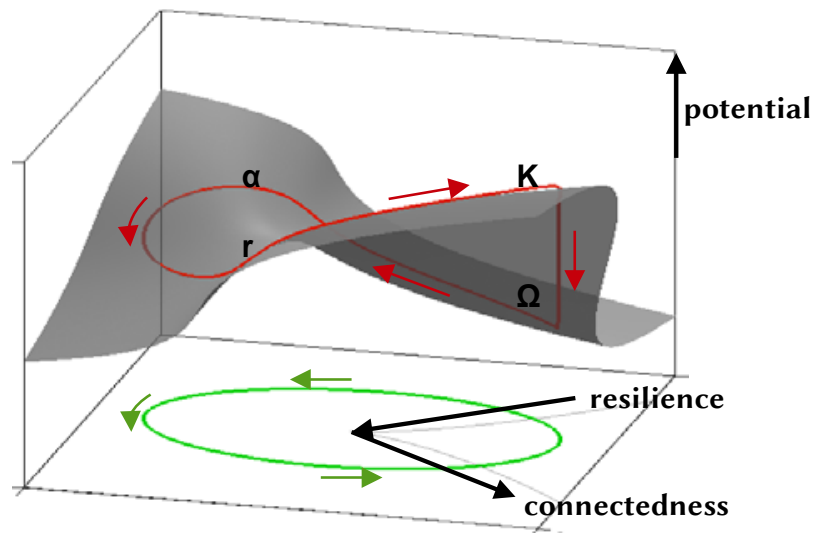

Figure 4: Adaptive cycle using a transformed cusp catastrophe. Compare this to the typical view of the adaptive cycle (Fig. 1).

\section{DISCUSSION}

As shown above, a cycle equivalent to the panarchy adaptive cycle can be constructed from the cusp catastrophe. This equivalence appears to be implicit in the adaptive cycle model and is approached but not explicitly stated at several points in Gunderson and Holling [1]. These authors refer to "catastrophe folds" in chapter 8 and show examples in Fig. 8-1 and 8-3 (these figures actually depict two-dimensional cross-sections of the cusp catastrophe). The rolling marble illustration in Fig. 8-3 is similar to a figure by Zeeman [17]. The equation given for alternative stable states in Appendix A resembles the cusp equilibria equation above. And the spruce budworm and forest ecosystem used to illustrate the adaptive cycle was first described using catastrophe theory by Jones [2] and by Ludwig, Jones and Holling [15].

Perhaps the creators of the adaptive cycle did not explicitly link it with the cusp catastrophe because of the critical backlash against catastrophe theory that occurred in the early 1980s, shortly after the theory was initially popularized with excessive claims and some inappropriate applications [18]. Much of the criticism leveled against the theory was a reaction to the fact that certain qualitative uses seemed to imply that these uses were rigorously based on Thom's findings. This criticism was often valid, since claims to rigorous use of catastrophe theory require technical justification. For example, one has to demonstrate that the dynamic system is a gradient system, that the phenomenon of interest occurs near the singularity, and that discontinuous effects depend on the specified number of control variables. But heuristic use of catastrophe theory can be justified, even if the validity of these assumptions is not demonstrated, if application of the theory is empirically tested. Use of the theory might even be justified if it is not assessed quantitatively but nonetheless offers a conceptually rich qualitative model of important features of the phenomenon of interest. What must not be done, however, is to claim that Thom's theory must apply, a priori, without demonstrating the validity of the theory's assumptions.

Formalizing the adaptive cycle with the cusp catastrophe does not prove that the adaptive cycle correctly applies to any particular ecological or human system. This is an empirical question that requires a separate investigation. (Admittedly, it is more difficult to test a topological model than one defined metrically, but catastrophe theoretic models have been subjected to empirical assessment. Attempting such an assessment here with data on a particular system is, however, beyond the scope of this paper.) Nor does formalization derive support from Thom's topological findings, since development of a cusp model of the adaptive cycle has not proceeded deductively. This paper merely suggests that the adaptive cycle can be cast into mathematical form, which adds specificity to qualitative uses of the model, and also opens up the possibility of quantitative testing. Because the adaptive cycle is general, any attempt to formalize it-via the cusp catastrophe or some other approachwill require some assumptions to be made. The assumptions in the present attempt include the orientation of the control variables and the trajectory of their values over time. But note that the elliptical trajectory assumed here isn't required; a circle or any roughly similar closed loop would work as well. (Representation of potential lost during the $\alpha$-phase to $r$-phase transition may also require a modification of the behavior surface.) These assumptions seem reasonable given the descriptions of the adaptive cycle provided by Gunderson and Holling, but as the adaptive cycle model evolves, other assumptions may be more appropriate. A cusp interpretation of the adaptive cycle may assist such further development.

The adaptive cycle has rich descriptive power in its iconic loop of two stages: the front loop of slow, predictable, and stable production and accumulation, and the back loop of fast, unpredictable, and unstable invention and reorganization. Formalization with the cusp catastrophe not only provides suitable mathematics for the standard four-phase adaptive cycle, it also accommodates modifications of this cycle. By constraining connectedness and resilience as shown in the above figures, the catastrophic crash in the transition from the K-phase to the $\Omega$-phase of the adaptive cycle is inevitable. But catastrophe theory leaves the trajectory of the control variables completely unspecified; the trajectory must be defined by the 
modeler who is using the theory. Thus formalizing the adaptive cycle via the cusp catastrophe does not imply that this crash is inevitable, and presentations of the adaptive cycle in [1] and elsewhere similarly state that the standard cycle is not inevitable. More specifically, control variable trajectories other than the one shown in Fig. 3(b) and Fig. 4 are possible. This path proposed in this paper was chosen to show how the cusp catastrophe can yield the adaptive cycle, but different trajectories might, for example, yield the "poverty trap" or the "rigidity trap" discussed in [1, p.95], both of which differ from the standard cycle. We have not tried to identify trajectories that produce such outcomes, but if such trajectories can be found, this would support the position of this paper that catastrophe theory is well suited to formalize the adaptive cycle. Trajectories that cause a jump in potential from the lower surface to the upper surface might even suggest novel solutions to sustainability efforts that would otherwise be overlooked.

\section{CONCLUSION}

Comparison of the panarchy adaptive cycle, a general model of change in natural and human systems, with the cusp catastrophe of catastrophe theory shows that the adaptive cycle can be formalized by the cusp catastrophe. While other ways of formalizing the adaptive cycle are possible, the widespread use of the cusp catastrophe in ecological modeling makes this a natural approach to formalization. By using the constrained control variables from the adaptive cycle as parameters in the behavior equation for the cusp catastrophe, a cycle very similar to the adaptive cycle can be constructed. Formalizing the panarchy adaptive cycle with the cusp catastrophe may provide direction for more rigorous and perhaps even more diverse applications of the adaptive cycle, thereby augmenting its usefulness in guiding sustainability efforts.

\section{ACKNOWLEDGMENTS}

We thank the anonymous reviewers for valuable suggestions.

\section{REFERENCES}

[1] Lance H. Gunderson and C.S. Holling. 2002. H

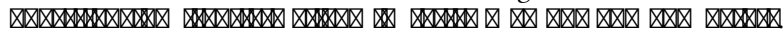
Island Press.

[2] Dixon D. Jones. 1977. Catastrophe theory applied to ecological systems. 29(1): 1-15; DOI: https://doi.org/10.1177/003754977702900102.

[3] Michael R. Rose and Rudolf Harmsen. 1981. Ecological outbreak dynamics and the cusp catastrophe.

[4] Max Rietkerk, Pieter Ketner, Leo Stroosnijder, and Herbert H.T. Prins. 1996. Sahelian rangeland development; a catastrophe? 》邓

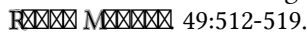

[5] L.E. Frelich and P.B. Reich. 1998. Disturbance severity and threshold responses in the boreal forest. 7. http://www.consecol.org/vol2/iss2/art7/

[6] H. Hesseln, D.B. Rideout, and P.N. Omi. 1998. Using catastrophe theory to model wildfire behavior and control. 28:852-862.

[7] Jason Matthiopoulos, Robert Moss, and Xavier Lambin. 2002. The kin facilitation hypothesis for red grouse population cycles: territorial dynamics of the family cluster.
291-307

[8] R. A. Washington-Allen, R. D. Ramsey, N. E.West, and R. A. Efroymson. 2006. A remote sensing-based protocol for assessing rangeland condition and trend. 59(1):19-29

[9] D. R. Lockwood and J. A. Lockwood. 2008. Grasshopper population ecology: catastrophe, criticality, and critique. 13(1):34. http://www.ecologyandsociety.org/vol13/iss1/art34/

[10] Steven D. Prager and William A. Reiners. 2009. Historical and emerging practices in ecological topology. $6: 160-171$

[11] Marten Scheffer. 2009. New Jersey: Princeton University Press.

[12] Marina Hirota et al. 2011. Global Resilience of Tropical Forest and Savanna to Critical Transitions. 334:232-234. DOI: $10.1126 /$ science. 1210657

[13] Marten Scheffer et al. 2012. Anticipating Critical Transitions. 338, 344-348; DOI: 10.1126/science. 1225244

[14] D. Ludwig, D.D. Jones and C.S. Holling. 1978. Qualitative Analysis of Insect Outbreak Systems: The Spruce Budworm and Forrest.

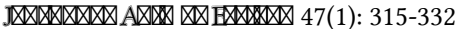

[15] C.S. Holling. 1981. Forest insects, forest fires, and resilience. Fire Regimes and Ecosystem Properties. U General Technical Report WO-26. Washington DC.

[16] R. Thom. 1975. $\triangle G \times \square$ W.A. Benjamin, Inc.

[17] E.C. Zeeman. 1977. Reading Mass.: Addison-Wesley.

[18] J.B. Rosser, Jr. 2007. The rise and fall of catastrophe theory applications in economics: Was the baby thrown out with the bathwater? 3280 . 